\title{
Multi-Pixel Ka-Band Radiometer for the QUIJOTE Experiment (Phase II)
}

\author{
Juan L. Cano, Enrique Villa, Jaime Cagigas, Beatriz Aja, \\ J. Vicente Terán, Ana R. Pérez, Luisa de la Fuente, \\ Eduardo Artal, Angel Mediavilla \\ Dep. Ingeniería de Comunicaciones \\ Universidad de Cantabria \\ 39005, Santander, Spain \\ juanluis.cano@unican.es
}

\begin{abstract}
This paper presents the configuration of the Ka-band radiometer developed for the Phase II of the QUIJOTE radio astronomy experiment, as well as the design of the different subsystems involved in the instrument. The new configuration, consisting of around 30 modified receivers working in the $26-36$ $\mathrm{GHz}$ band, avoids the need of a rotating polar modulator at cryogenic temperatures, which is a source of mechanical and thermal difficulties. Moreover, the larger number of receivers will increase the instrument sensitivity. These two aspects are a clear advantage over the receiver developed for the experiment Phase I. The present paper also gives detailed information of some designed subsystems such as the feedhorn, the polarizer, the orthomode transducer, the cryogenic low-noise amplifiers and the back-end module.
\end{abstract}

Keywords-cryogenic amplifiers; polarimeter; radiometer; radio astronomy; very low-noise receivers; waveguides

\section{INTRODUCTION}

The QUIJOTE experiment is a radio astronomy project aimed to characterize the polarization of the Cosmic Microwave Background (CMB) and other galactic and extragalactic emission processes [1]. This experiment is conceived as a two phase project. In the first one, phase I, an instrument with five pixels covering different bands in the $10-$ $30 \mathrm{GHz}$ range is designed [2]-[4]. This phase has recently started its observing stage and the first scientific results are expected to be published soon from the collected data. The second phase, which is under development right now, foresees the design of a new instrument with a large number of pixels or receivers, around 30, all of them working in the bandwidth from 26 to $36 \mathrm{GHz}$, where the final number depends on the feedhorn outer diameter and the focal plane dimension.

\author{
Roger Hoyland \\ Instituto de Astrofísica de Canarias \\ 38205, La Laguna, Tenerife, Spain \\ rjh@iac.es
}

In the first instrument there is only one pixel working in the 26 to $36 \mathrm{GHz}$ band. This receiver, conceived as a demonstrator for the second instrument, is based on a rotating polar modulator which enables the measurement of three Stokes parameters (Q, U and I) simultaneously and, at the same time, overcomes the $1 / \mathrm{f}$ noise problems in the receiver when it is spun at high speed. Due to the spinning difficulties of the polar modulator in a cryogenic environment encountered during the first instrument development, the second instrument receiver configuration was modified in order to remove the rotating dependence of the system. The replacement of the rotating polar modulator by a fixed polarizer involves the modification of the receiver configuration in order to maintain the instrument functionality. Fig. 1 shows the new configuration, which includes a fixed polarizer and $90^{\circ}$ - and $180^{\circ}$-phase switches to generate four polarization states to minimize $1 / \mathrm{f}$ noise and different systematics in the receiver.

This paper presents the development of different hardware designed for the second instrument, which improve the performance regarding their counterparts in the first instrument as well as the performance of the instrument itself.

\section{SUBSySTEMS DESIGN}

\section{A. Feedhorn}

The antenna of each pixel is a corrugated feedhorn, whose matching and cross-polarization characteristics ared defined by its throat profile. In the first instrument, the circular input waveguide diameter is $7.1 \mathrm{~mm}$ which easily fits to the WR28 dimension; however, it was found that this diameter was not large enough to enable a good performance at the lower end of

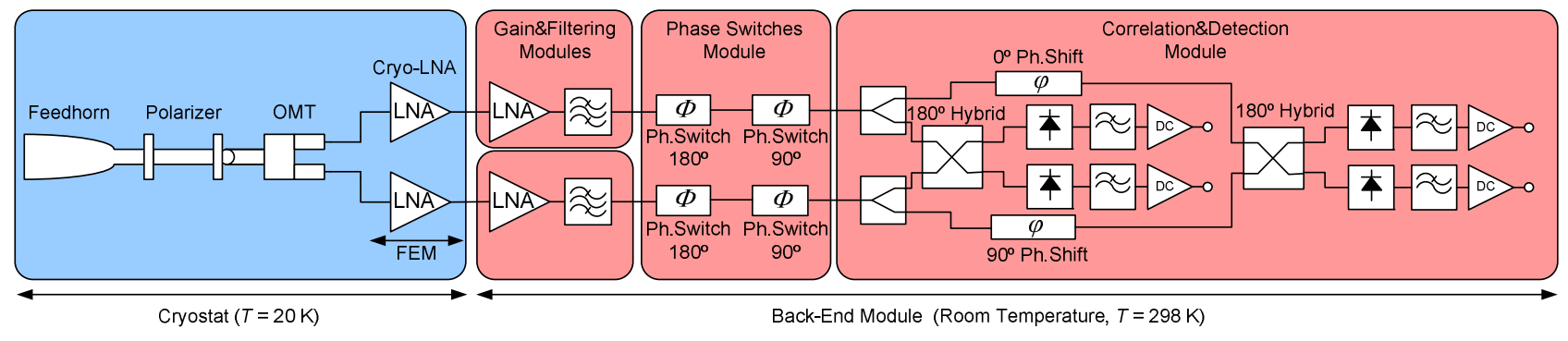

Figure 1. Configuration of each receiver in the QUIJOTE experiment (phase II).

This work has been funded by the Ministerio de Ciencia e Innovación (Spain) under Astronomy and Astrophysics research programme, reference AYA2010-21766-C03-03, and the FPI grant BES-2011-046199. 
the bandwidth, especially in the matching parameter. Therefore, for the second instrument, this diameter has been increased to $8 \mathrm{~mm}$ and the whole throat has been re-designed accordingly, optimizing its performance for the best crosspolarization possible, which is the most important parameter for an antenna intended for polarimetry. The remaining horn shape is designed and optimized for achieving a good directivity and radiation pattern. Fig. 2 shows performance measurements of the feedhorn.

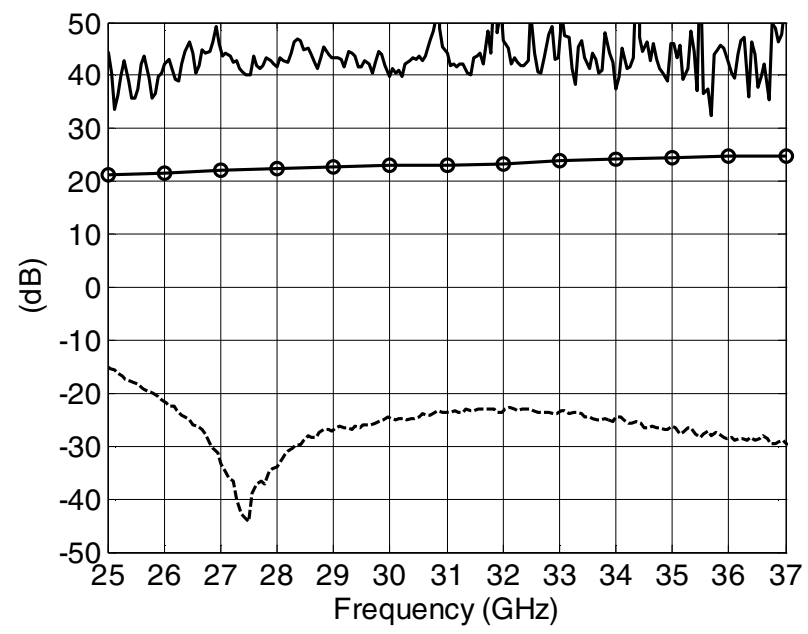

Figure 2. Feedhorn measured performance; return loss (dashed line); directivity (circles); and cross-polarization (solid line). Cross-polarization result is affected by the noise floor in the measurement setup.

\section{B. Polarizer}

The CMB is believed to be a nearly unpolarized signal with a residual linear polarization $(<7 \%)$. This linear polarization needs to be converted into circular polarization in the receiver. Usually, this is accomplished using a septum polarizer, but this component is difficult to design for relative bandwidths greater than $30 \%$. For this reason, in this instrument it was decided to design a polarizer and an orthomode transducer. If the polarizer is rotated $45^{\circ}$ to the orthomode, then both components together work as a septum polarizer. The polarizer designed for this instrument is a scaled and mechanically improved version of the circuit presented in [5]. Fig. 3 shows the measured characteristics of the polarizer.

\section{Orthomode Transducer}

Following the polarizer, an orthomode transducer (OMT) separates the orthogonal components of the incoming signal. These orthogonal components, after being amplified and conveniently correlated and detected, enable to calculate the so-called Stokes parameters (three out of four), and therefore to obtain the signal polarization state.

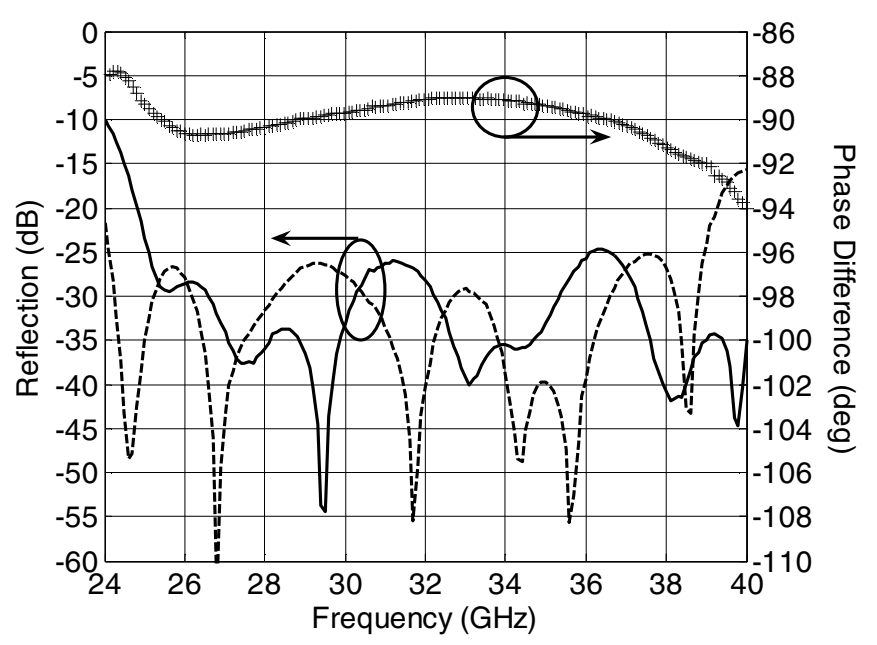

Figure 3. Measured characteristics of the polarizer; reflection of the two propagated modes in the square waveguide, $\mathrm{TE}_{10}$ and $\mathrm{TE}_{01}$ (solid and dashed lines); phase difference between the propagated modes (crosses). The phase difference is within $\pm 1^{\circ}$ in the band of interest.

The OMT design is based on the same subsystem developed for the first instrument [6]. Small modifications were carried out during the manufacture process in order to minimize misalignment problems in the scatterer and consequently, to improve the OMT performance. Fig. 4 shows the measured characteristics of the OMT. The isolation between rectangular ports is, in general, below $-50 \mathrm{~dB}$.

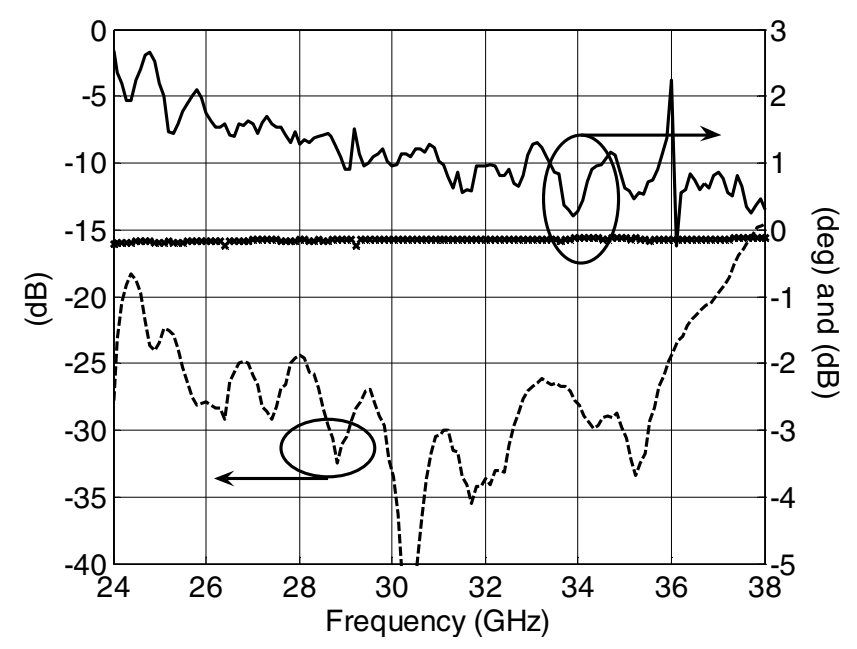

Figure 4. Orthomode transducer performance; reflection at the square port in dashed line (dB); phase difference between OMT branches in solid line (deg); and transmission with crosses $(\mathrm{dB})$, mean value across the band is $-0.15 \mathrm{~dB}$.

A picture of the waveguide components presented here and assembled in the laboratory is shown in Fig. 5. 


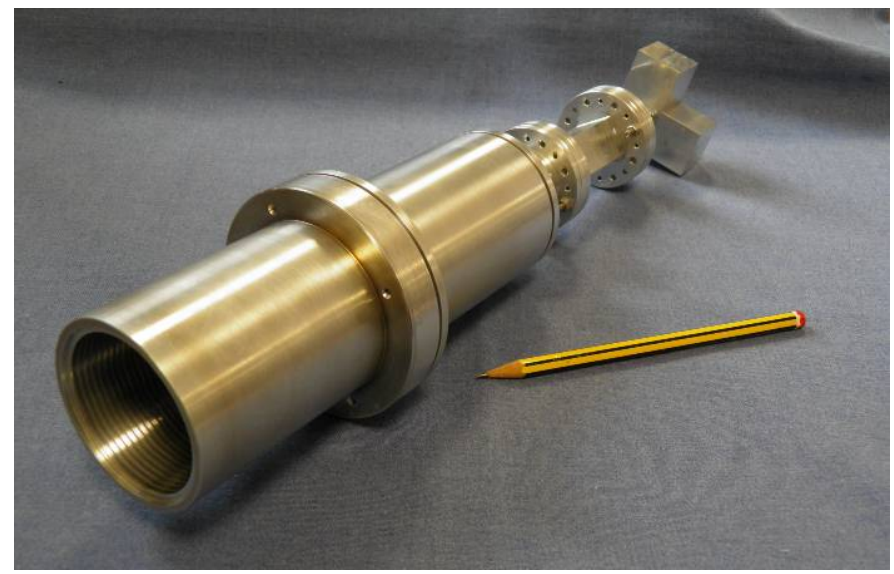

Figure 5. Picture of the feedhorn, the polarizer and the orthomode transducer assembled in the laboratory.

\section{Cryogenic Low-Noise Amplifiers}

One of the most important subsystems in the receiver chain is the first low-noise amplifier (LNA), since it has a great impact on the receiver sensitivity. In order to increase this sensitivity, the LNA needs to have a high gain and the lowest noise possible. For this reason, this amplifier is cooled at cryogenic temperatures.

For the second instrument a new cryogenic low-noise amplifier has been designed. It combines the hybrid and monolithic technologies. The first stage is a $4 \times 15 \mu \mathrm{m}$ discrete transistor from the IAF (Freiburg, Germany) manufactured in its $50 \mathrm{~nm}$ mHEMT process. The second part in the LNA is a monolithic 4-stage MMIC LNA specifically designed using the D01MH process from OMMIC foundry (France). Although the LNA is fully designed using mHEMT GaAs technology, it provides a good noise result as it is depicted in Fig. 6, not very far from similar designs using InP technology [7]. The dc power consumption of the whole amplifier is only $15.1 \mathrm{~mW}$.

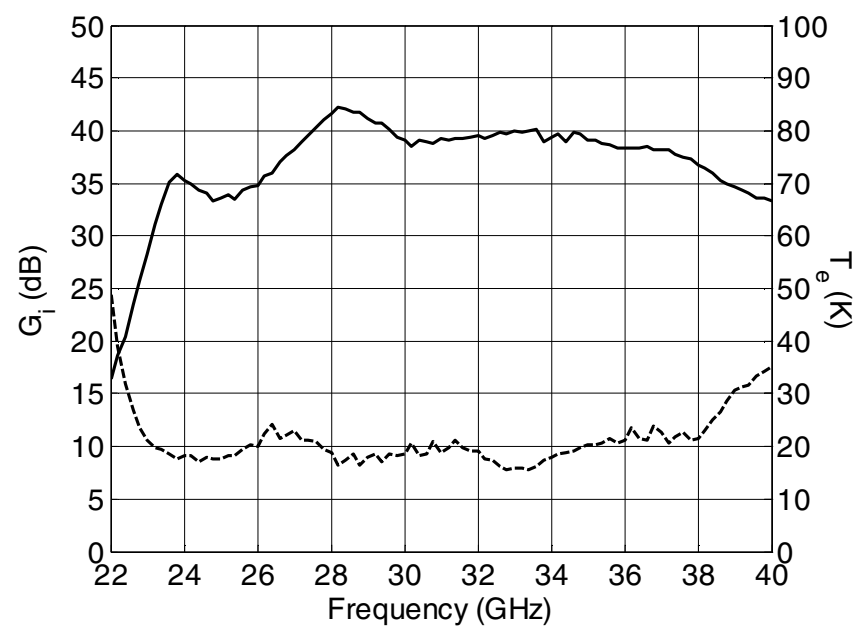

Figure 6. Measured results of the LNA at $16 \mathrm{~K}$ ambient temperature; insertion gain (solid line) with a mean value of $G_{i}=39.4 \mathrm{~dB}$ across the band of interest; and noise temperature (dashed line) with a mean value of $T_{e}=19.1$ $\mathrm{K}$ across the band of interest.
Fig. 7 shows a picture of the designed cryogenic LNA with the cover removed.

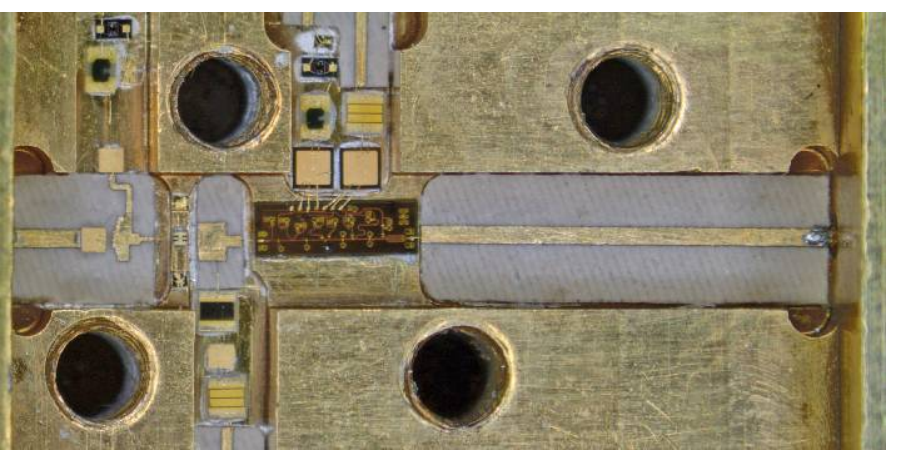

Figure 7. Picture of the designed cryogenic LNA. External dimensions are $35 \times 20 \times 10 \mathrm{~mm} 3$, excluding connectors.

\section{E. Back-End Module}

The back-end module (BEM) provides further amplification, band filtering, electronic switching to generate all the polarization states, signal correlation, and detection. In order to implement all these functions and to test them, the back-end module is divided in three modules: the first one contains the low-noise amplifiers and band-pass filter; the second one includes the $180^{\circ}$ - and $90^{\circ}$-phase switches; in the last module the $180^{\circ}$-hybrids, detectors and dc amplifiers are assembled.

From the four BEM outputs the three Stokes parameters (Q, $\mathrm{U}$ and I) can be directly obtained and therefore the polarization of the incoming signal can be calculated. Phase switches enable to obtain the Stokes parameters from different outputs, which minimizes the effect of systematics in the receiver.

The BEM amplification is carried out using commercial LNAs since the noise requirements are not as critical as in the front-end module (FEM). The model AMMC-6241 from Avago Technologies was selected for this purpose. The bandpass filter is designed using microstrip technology on alumina substrate. Design information of this filter was described in [4]. A picture of the gain and filtering module is shown in Fig. 8.

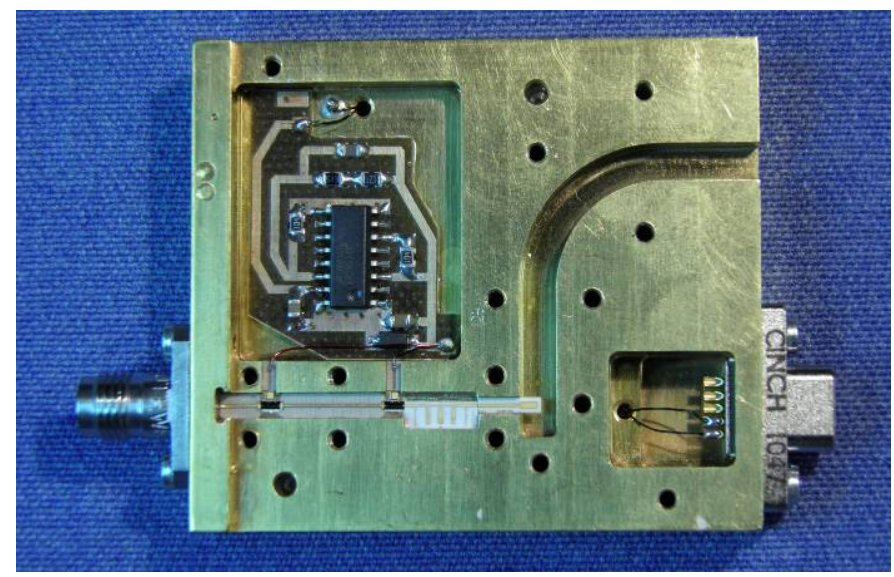

Figure 8. View of the gain and filtering block in the back-end module. 
The phase switches module is currently under development. It contains two $180^{\circ}$-phase switches and two $90^{\circ}$-phase switches in order to produce the four required states $\left(0^{\circ}, 90^{\circ}\right.$, $180^{\circ}$ and $270^{\circ}$ ) in each branch. The $180^{\circ}$-phase switch has been designed on alumina substrate with PIN diodes following the scheme presented in [8].

For the signals correlation a $180^{\circ}$-hybrid is designed on alumina substrate. The configuration selected for this component is an optimized circuit based on [9], [10]. Measurement results show $\pm 5^{\circ}$ phase error within the band, with $0.5 \mathrm{~dB}$ maximum amplitude imbalance, isolation better than $25 \mathrm{~dB}$ and return losses better than $10 \mathrm{~dB}$ in all the ports for this hybrid.

In order to obtain the Stokes parameters, it is necessary to include an additional $90^{\circ}$ phase shift in one BEM branch. For this instrument, two phase shifter configurations were manufactured in waveguide technology and measured: a corrugations-based phase shifter, and a stubs-based phase shifter. It was found that the corrugations-based circuit was more robust against mechanical tolerances and therefore it was finally implemented in the BEM. This $90^{\circ}$-phase shifter showed $\pm 3^{\circ}$ phase error with a return loss better than $25 \mathrm{~dB}$ in the band of interest. Finally, the signal detection is carried out with a Schottky diode detector designed on alumina substrate already reported in [4]. Fig. 9 shows a picture of the correlation and detection block.

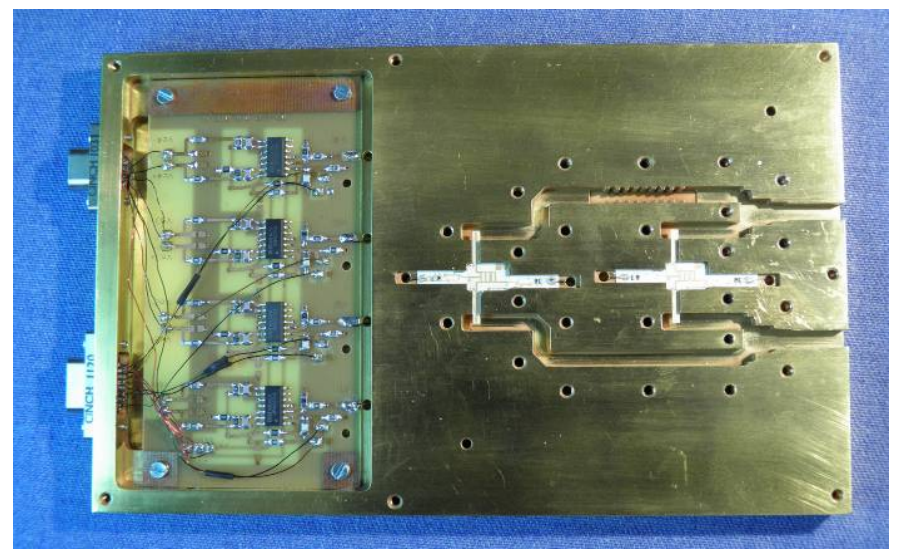

Figure 9. Picture of the correlation module in the BEM. The corrugationsbased $90^{\circ}$-phase shifter can be easily identified in the upper waveguide. The de circuitry in the left side is the differential video amplifiers for the outputs.

\section{CONCLUSIONS}

This paper has presented the new receiver configuration for the Phase II of the QUIJOTE experiment. This new instrument configuration overcomes some problems arisen during the development of the experiment Phase I and increases the receiver sensitivity with a simpler design.

Moreover, detailed information of the design and performance of different receiver subsystems is provided. All the subsystems show an excellent behavior in the whole band of interest, from 26 to $36 \mathrm{GHz}$. Once assembled, the receiver is expected to have an excellent performance and therefore to be able to achieve the experiment goals.

\section{ACKNOWLEDGMENT}

Authors thank Eva Cuerno and Santiago Cembellín for their assembly and mechanical work during the current project.

\section{REFERENCES}

[1] J.A. Rubiño-Martin et al., "The QUIJOTE CMB Experiment", in Highlights of Spanish Astrophysics V, Astrophysics and Space Science Proceedigns, Part 3, Ed. Springer, 2010, pp. 127-135.

[2] J. L Cano et al., "The Ka-Band Receiver for the QUIJOTE Experiment", Proc. 6th European Microwave Integrated Circuits Conf. (6th EuMIC), Manchester, UK, Oct. 2011, pp. 620-623.

[3] E. Artal et al., "Cosmic microwave background polarization receivers: QUIJOTE experiment", Proc. 40th European Microwave Conference, 28-30 September, Paris, 2010. pp. 497-500.

[4] J. L. Cano, B. Aja, E. Villa, L. de la Fuente, and E. Artal, "Broadband Back-End Module for Radio-Astronomy Applications in the Ka-Band", Proc. 38th European Microwave Conference, Oct. 2008, Amsterdam. pp. 1113-1116.

[5] A. Tribak, A. Mediavilla, J. L Cano, M. Boussouis, and K. Cepero, "Ultra-Broadband Low Axial Ratio Corrugated Quad-Ridge Polarizer", Proc. 39th European Microwave Conf. (39th EuMC), Roma, Italy, Oct. 2009, pp. 73-76.

[6] J. L. Cano, A. Tribak, R. Hoyland, A. Mediavilla, and E. Artal, "Full Band Waveguide Turnstile Junction Orthomode Transducer with Phase Matched Outputs", Int. Jrnl. RF and Microwave CAE, vol. 20, no. 3, May 2010, pp. 333-341.

[7] Y-L. Tang, N. Wadefalk, M. A. Morgan, and S. Weinreb, "Full Ka-Band High Performance InP MMIC LNA Module", IEEE MTT-S Digest, San Francisco, USA, June 2006, pp. 81-84.

[8] E. Villa, B. Aja, J. L. Cano, L. de la Fuente, and E. Artal, "Cryogenic Ka-Band $180^{\circ}$ Phase Switch Based on Schottky Diodes", IEEE Microwave and Wireless Comp. Lett., vol. 22, no. 2, Feb. 2012, pp. 5254.

[9] M. Muraguchi, T. Yukitake, and Y. Naito, "Optimum Design of $3 \mathrm{~dB}$ Branch-line Couplers Using Microstrip Lines", IEEE Trans. Microwave Theory and Tech., vol. MTT-31, no. 8, Aug. 1983, pp. 674-678.

[10] R. J. Hoyland, “A New MMIC, Wideband $180^{\circ}$ Phase Switch Design for Millimeter Wave Applications", Proc. $3^{\text {rd }}$ ESA Workshop on Millimeter Wave Technology and Applications, Espoo, Finland, May 2003, pp. 305-310. 